hep-th/9602154

YCTP-P $4 / 96$

RU-96-10

\title{
Counting Curves with Modular Forms
}

\author{
Måns Henningson and Gregory Moore* \\ Department of Physics \\ Yale University \\ P. O. Box 208120 \\ New Haven, CT 06520-8120, USA \\ mans@genesis5.physics.yale.edu \\ moore@castalia.physics.yale.edu
}

\begin{abstract}
We consider the type IIA string compactified on the Calabi-Yau space given by a degree 12 hypersurface in the weighted projective space $\mathbf{P}_{(1,1,2,2,6)}^{4}$. We express the prepotential of the low-energy effective supergravity theory in terms of a set of functions that transform covariantly under $P S L(2, \mathbb{Z})$ modular transformations. These functions are then determined by monodromy properties, by singularities at the massless monopole point of the moduli space, and by $S \leftrightarrow T$ exchange symmetry.
\end{abstract}

February 26, 1996; revised April 30, 1996.

* Currently visiting Rutgers Dept. of Physics. 


\section{Introduction}

Heterotic/Type II string duality has focused attention on the special Kähler geometry of vectormultiplets as a means of defining some nonperturbative effects in the heterotic string [1] [2].

In this note we describe one means for obtaining the prepotential for IIA vectormultiplet geometry using properties of modular forms. In particular we focus on the model described in [2] based on IIA compactification on a manifold $X(1,1,2,2,6)$ with $\left(h^{1,1}, h^{2,1}\right)=(2,128)$. In this example the Kähler cone has two coordinates $S, T$ and the special Kähler coordinate $S$ can be identified with the heterotic dilaton [2] [3]. Therefore, on the heterotic side, nonrenormalization theorems show that the (inhomogeneous) vectormultiplet prepotential has the general form:

$$
\mathcal{F}(S, T)=S T^{2}+f_{0}(T)+\sum_{k=1}^{\infty} f_{k}(T) e^{2 \pi i k S}
$$

while on the type IIA side we have:

$$
\mathcal{F}(S, T)=S T^{2}+r(T)+\frac{1}{(2 \pi i)^{3}} \sum_{j, k} n_{j, k} L i_{3}\left(e^{2 \pi i(j T+k S)}\right)
$$

where $L i_{3}$ is the trilogarithm, $n_{j, k}$ counts rational curves in $X(1,1,2,2,6)$ and $r(T)$ is a cubic polynomial.

The prepotential (1.2) in this model has been computed in [4] [5] using mirror symmetry. In this paper we suggest another method - based on monodromy and singularity structure - by which one can determine the prepotential. The procedure may be viewed as a generalization of the method used in [6] [7] [8] to determine the one-loop prepotential $f_{0}(T)$ for this model.

In brief, we use the nonperturbative monodromy of the special Kähler periods determined in [9] to find a set of transformation laws for the prepotential. The monodromy group of [9] is a discrete subgroup of $S p(6 ; \mathbb{Z})$. It acts on the Kähler cone, and, in the

limit $q_{2}=e^{2 \pi i S} \rightarrow 0$ the action reduces to the standard Möbius action of $P S L(2, \mathbb{Z})$ on $T$. Therefore, one may expect the functions $f_{k}(T)$ to be related to modular forms for $P S L(2, \mathbb{Z})$. We find that this is indeed so. More precisely, we can make an upper triangular transformation of differential polynomials from $f_{k}(T)$ to a new set of functions $h_{k}(T)$ which are modular forms. This transformation is summarized in equations (3.1) - (3.4) below. The relation of the prepotential to 
modular forms has also been investigated in [10].

Assuming the singularity structure at $T=i$ implied by the connection [2] [9] to the Seiberg-Witten massless monopole singularity we find that $h_{k}(T)$ can be written in terms of polynomials of Eisenstein series. Finally, using the $T \leftrightarrow S$ symmetry implied by $n_{j, k}=$ $n_{j, j-k}$ [5] we find that the polynomial in Eisenstein series is uniquely determined. The $T \leftrightarrow S$ symmetry has been the subject of much recent discussion [11] [12].

We must emphasize that the crucial "upper triangular" transformation was discovered "experimentally" using a computer and we have not proved it analytically, although it has been checked extensively.

We hope that this work might offer an alternative method to the standard mirror symmetry techniques for counting rational curves in (some) Calabi-Yau manifolds,

which might be of interest in multiparameter examples. A problem with this approach, though, is that one would first have to determine the monodromies.

More speculatively, our methods should help to determine root supermultiplicities of generalized Kac-Moody superalgebras [13] [14] [15] [16].

\section{The monodromy action}

We will consider the type IIA string compactified on the Calabi-Yau threefold given by a degree 12 hypersurface in the weighted projective space $\mathbf{P}_{(1,1,2,2,6)}^{4}$ discussed in [2] [4] [5]. The degrees of freedom of the low-energy supergravity theory are described by three vector superfields $X^{0}, X^{1}$ and $X^{2}$, corresponding to the graviphoton and two abelian YangMills multiplets respectively. Their dynamics are governed by a holomorphic prepotential $F\left(X^{0}, X^{1}, X^{2}\right)$. To get the correct number of propagating fields, $F$ must be homogeneous

of degree two in the $X^{i}$ [17. It is convenient to introduce the inhomogeneous special coordinates $S$ and $T$, which are defined in terms of the homogeneous coordinates $X^{0}, X^{1}$ and $X^{2}$ as $S=X^{1} / X^{0}$ and $T=X^{2} / X^{0}$. The prepotential can then be written as

$$
F\left(X^{0}, X^{1}, X^{2}\right)=\left(X^{0}\right)^{2}\left(S T^{2}+f(S, T)\right)
$$

where the first term arises at tree-level and the second term encodes all (perturbative and non-perturbative) quantum corrections. 
We define the periods $F_{i}$ for $i=0,1,2$ as $F_{i}=\frac{\partial}{\partial X^{i}} F$, i.e.

$$
\begin{aligned}
& F_{0}=X^{0}\left(-S T^{2}+2 f-S \frac{\partial f}{\partial S}-T \frac{\partial f}{\partial T}\right) \\
& F_{1}=X^{0}\left(T^{2}+\frac{\partial f}{\partial S}\right) \\
& F_{2}=X^{0}\left(2 S T+\frac{\partial f}{\partial T}\right),
\end{aligned}
$$

and assemble the homogeneous coordinates and the periods in a period vector $\Pi$ given by $\Pi=\left(\begin{array}{llllll}X^{0} & X^{1} & X^{2} & F_{0} & F_{1} & F_{2}\end{array}\right)^{T}$. As we encircle a singular divisor in the moduli space, the period vector $\Pi$ is acted on by multiplication by an element of the monodromy group. This group is a subgroup of $S p(6, \mathbf{Z})$ generated by three elements $S_{1}, T_{1}$ and $T_{2}$ [9], which in our basis are given by

$$
\begin{aligned}
S_{1} & =\left(\begin{array}{cccccc}
0 & 0 & 0 & 0 & -1 & 0 \\
-1 & 0 & 0 & 1 & -1 & 0 \\
0 & 0 & 1 & 0 & 0 & 0 \\
1 & 1 & 0 & 0 & 1 & 0 \\
-1 & 0 & 0 & 0 & 0 & 0 \\
0 & 0 & 0 & 0 & 0 & 1
\end{array}\right) \\
T_{1} & =\left(\begin{array}{cccccc}
1 & 0 & 0 & 0 & 0 & 0 \\
0 & 1 & 0 & 0 & 0 & 0 \\
1 & 0 & 1 & 0 & 0 & 0 \\
-5 & -1 & -4 & 1 & 0 & -1 \\
1 & 0 & 2 & 0 & 1 & 0 \\
0 & 2 & 4 & 0 & 0 & 1
\end{array}\right) \\
T_{2} & =\left(\begin{array}{ccccccc}
1 & 0 & 0 & 0 & 0 & 0 \\
1 & 1 & 0 & 0 & 0 & 0 \\
0 & 0 & 1 & 0 & 0 & 0 \\
0 & 0 & 0 & 1 & -1 & 0 \\
0 & 0 & 0 & 0 & 1 & 0 \\
0 & 0 & 2 & 0 & 0 & 1
\end{array}\right) .
\end{aligned}
$$

The monodromies under $T_{1}, T_{2}$ can be deduced, on the type II side, from the monodromy of the mirror manifold at $\infty$ in complex structure space. The monodromy under $S_{1}$ must then be deduced, on the type II side, by solving Picard-Fuchs equations. Alternatively, assuming string/string duality, it may be deduced, on the heterotic side, from the one-loop approximation to $f(S, T)$.

We will now determine the action of the monodromy group on the special coordinates $S$ and $T$ and the function $f$ appearing in the prepotential (2.1). It follows from the above 
that the $T_{2}$ transformation acts as

$$
\begin{aligned}
& S \rightarrow S+1 \\
& T \rightarrow T \\
& f \rightarrow f .
\end{aligned}
$$

This means that $f$ may be expanded in powers of $q_{2}=\exp 2 \pi i S$ [18], i.e.

$$
f(S, T)=\sum_{k=0}^{\infty} q_{2}^{k} f_{k}(T)
$$

for some functions $f_{k}$. The $T_{1}$ transformation acts as

$$
\begin{aligned}
& S \rightarrow S \\
& T \rightarrow T+1 \\
& f \rightarrow f+2 T^{2}-\frac{5}{2} .
\end{aligned}
$$

In terms of the functions $f_{k}$ in (2.5), this means that

$$
f_{0}(T+1)=f_{0}(T)+2 T^{2}-\frac{5}{2}
$$

and

$$
f_{k}(T+1)=f_{k}(T), \quad k>0 .
$$

The consequences of the $S_{1}$ transformation are less straightforward to extract. It acts as

$$
\begin{aligned}
& S \rightarrow \tilde{S}=1+\frac{S+T^{-2}-2 T^{-2} f+S T^{-2} \frac{\partial f}{\partial S}+T^{-1} \frac{\partial f}{\partial T}}{1+T^{-2} \frac{\partial f}{\partial S}} \\
& T \rightarrow \tilde{T}=-\frac{T^{-1}}{1+T^{-2} \frac{\partial f}{\partial S}}
\end{aligned}
$$

and

$$
f \rightarrow \frac{T^{-4} f-\frac{1}{2} T^{-4}-T^{-2}-\frac{1}{2}+\ldots}{\left(1+T^{-2} \frac{\partial f}{\partial S}\right)^{3}}
$$

where the omitted terms are proportional to powers of $\frac{\partial f}{\partial S}$. Inserting (2.5) and (2.9) in (2.10) and taking the $S \rightarrow i \infty$ limit, we find that

$$
f_{0}(-1 / T)=T^{-4} f_{0}(T)-\frac{1}{2} T^{-4}-T^{-2}-\frac{1}{2} .
$$


The transformation properties of the functions $f_{k}$ for $k>0$ are most easily deduced by considering $\frac{\partial f}{\partial S}$, which transforms under the $S_{1}$ transformation as

$$
\frac{\partial f}{\partial S} \rightarrow \frac{T^{-4} \frac{\partial f}{\partial S}}{\left(1+T^{-2} \frac{\partial f}{\partial S}\right)^{2}} .
$$

Inserting (2.5) and (2.9) in (2.12), we get

$$
\sum_{k=1}^{\infty} 2 \pi i k f_{k}(\tilde{T}) \exp 2 \pi i k \tilde{S}=\frac{T^{-4} \sum_{k=1}^{\infty} 2 \pi i k f_{k}(T) \exp 2 \pi i S}{\left(1+T^{-2} \sum_{k=1}^{\infty} 2 \pi i k f_{k}(T) \exp 2 \pi i S\right)^{2}}
$$

where $\tilde{S}$ and $\tilde{T}$ are given in (2.9). By expanding this equation in powers of $q_{2}=\exp 2 \pi i S$ and expanding the functions $f_{k}$ on the left hand side in a Taylor series around $-1 / T$, we can recursively determine the transformation laws of these functions. For example, for $f_{1}$ and $f_{2}$ we get

$$
\begin{array}{rl}
f_{1}(-1 / T)=\exp 2 \pi & i\left(-T^{-2}+2 T^{-2} f_{0}(T)-T^{-1} f_{0}^{(1)}(T)\right) T^{-4} f_{1}(T) \\
f_{2}(-1 / T)=\exp 4 & i i\left(-T^{-2}+2 T^{-2} f_{0}(T)-T^{-1} f_{0}^{(1)}(T)\right) \\
\times & \left(T^{-4} f_{2}(T)\right. \\
& +T^{-5}\left(-2 \pi i f_{1}(T) f_{1}^{(1)}(T)\right) \\
& +T^{-6}\left(4 \pi i f_{1}(T) f_{1}(T)-2 \pi^{2} f_{1}(T) f_{1}(T) f_{0}^{(2)}(T)\right) \\
& +T^{-7} 4 \pi^{2} f_{1}(T) f_{1}(T) f_{0}^{(1)}(T) \\
& \left.+T^{-8}\left(2 \pi^{2} f_{1}(T) f_{1}(T)-4 \pi^{2} f_{0}(T) f_{1}(T) f_{1}(T)\right)\right)
\end{array}
$$

where superscripts in parenthesis indicate derivatives with respect to $T$.

\section{Transformation to modular forms}

The transformation laws (2.7), (2.8), (2.11) and (2.14) indicate that the functions $f_{k}$

are closely related to modular forms. Indeed, it follows from (2.7) and (2.11) that $f_{0}^{(5)}$ is a modular form of weight 6 [6] [19].

To see how the $f_{k}$ for $k>0$ are related to modular forms, we first introduce a new formal expansion parameter $q$ ( distinct from $q_{1}=\exp 2 \pi i T$ and $q_{2}=\exp 2 \pi i S$ ) and a function $h$ with an expansion of the form

$$
h=\sum_{k=0}^{\infty} q^{k} h_{k}(T) .
$$


Next, we define a set of functions $g_{(m)}$ for $m=0,1, \ldots$ recursively by

$$
\begin{aligned}
g_{(0)} & =\frac{\partial}{\partial S} f \\
g_{(m+1)} & =\sum_{n=0}^{\infty} \frac{1}{n !} \frac{1}{m n+1}\left(\frac{(2 m-1)(-1)^{m}}{6(2 m) !} h^{(2 m+2)}\right)^{n} \frac{\partial^{n}}{\partial S^{n}}\left(g_{(m)}\right)^{m n+1},
\end{aligned}
$$

where again superscripts in parenthesis denote derivatives with respect to $T$. Finally, we define the relationship between the functions $h$ and $f$ to be given by the equation

$$
h=\left.\lim _{m \rightarrow \infty}\left(\frac{\partial}{\partial S}\right)^{-1} g_{(m)}\right|_{q_{2}=q}
$$

where the integration constant arising from the $\left(\frac{\partial}{\partial S}\right)^{-1}$ operator is fixed by the requirement that the $q$ independent term of the right-hand side of 3.3 equal $f_{0}(T)$. Our conjecture is now that the $h_{k}(T)$ for $k>0$ transform covariantly with weight -4 under the modular group:

$$
\begin{aligned}
& h_{k}(T+1)=\exp \left(\frac{2 \pi i k}{3}\right) h_{k}(T) \\
& h_{k}(-1 / T)=\exp \left(\frac{2 \pi i k}{3}\right) T^{-4} h_{k}(T) .
\end{aligned}
$$

We have no analytic proof of this statement, but it has been checked by computer up to $k=7$.

The explicit formulae for the $h_{k}(T)$ can be obtained as follows. We begin by expanding (3.2) to get:

$$
\begin{aligned}
g_{(0)} & =\sum_{k=1}^{\infty} q_{2}^{k} 2 \pi i k f_{k} \\
g_{(1)} & =\sum_{k=1}^{\infty} q_{2}^{k} \exp \left(-\frac{\pi i k}{3} h^{(2)}\right) 2 \pi i k f_{k} \\
g_{(2)} & =\sum_{k=1}^{\infty} q_{2}^{k} \exp \left(-\frac{\pi i k}{3} h^{(2)}\right) \sum_{n=0}^{\infty} \frac{1}{(n+1) !}\left(-\frac{\pi i k}{6} h^{(4)}\right)^{n} \\
& \quad \times \sum_{k_{0}, \ldots, k_{n}=1}^{\infty} \delta_{k_{0}+\ldots+k_{n}, k} \prod_{i=0}^{n} 2 \pi i k_{i} f_{k_{i}}
\end{aligned}
$$


Then, inserting (3.1) in (3.3) and solving recursively for the $f_{k}$ in terms of the $h_{k}$, we get

$$
\begin{aligned}
& f_{0}=h_{0} \\
& f_{1}=\exp \left(\frac{\pi i}{3} h_{0}^{(2)}\right) h_{1} \\
& f_{2}=\exp \left(\frac{2 \pi i}{3} h_{0}^{(2)}\right)\left(h_{2}+\frac{\pi i}{3} h_{1} h_{1}^{(2)}-\frac{\pi^{2}}{6} h_{1} h_{1} h_{0}^{(4)}\right) \\
& f_{3}=\exp \left(\frac{3 \pi i}{3} h_{0}^{(2)}\right)\left(h_{3}+\frac{2 \pi i}{3} h_{2} h_{1}^{(2)}-\frac{\pi^{2}}{6} h_{1} h_{1}^{(2)} h_{1}^{(2)}+\frac{\pi i}{3} h_{1} h_{2}^{(2)}\right. \\
& -\frac{2 \pi^{2}}{3} h_{1} h_{2} h_{0}^{(4)}-\frac{2 \pi^{3} i}{9} h_{1} h_{1} h_{1}^{(2)} h_{0}^{(4)}+\frac{\pi^{4}}{18} h_{1} h_{1} h_{1} h_{0}^{(4)} h_{0}^{(4)} \\
& \left.-\frac{\pi^{2}}{6} h_{1} h_{1} h_{1}^{(4)}+\frac{\pi^{3} i}{18} h_{1} h_{1} h_{1} h_{0}^{(6)}\right) \\
& f_{4}=\exp \left(\frac{4 \pi i}{3} h_{0}^{(2)}\right)\left(h_{4}+\pi i h_{3} h_{1}^{(2)}-\frac{4 \pi^{2}}{9} h_{2} h_{1}^{(2)} h_{1}^{(2)}-\frac{8 \pi^{3} i}{81} h_{1} h_{1}^{(2)} h_{1}^{(2)} h_{1}^{(2)}\right. \\
& +\frac{2 \pi i}{3} h_{2} h_{2}^{(2)}-\frac{4 \pi^{2}}{9} h_{1} h_{1}^{(2)} h_{2}^{(2)}+\frac{\pi i}{3} h_{1} h_{3}^{(2)}-\frac{2 \pi^{2}}{3} h_{2} h_{2} h_{0}^{(4)} \\
& -\pi^{2} h_{1} h_{3} h_{0}^{(4)}-\frac{10 \pi^{3} i}{9} h_{1} h_{2} h_{1}^{(2)} h_{0}^{(4)}+\frac{13 \pi^{4}}{54} h_{1} h_{1} h_{1}^{(2)} h_{1}^{(2)} h_{0}^{(4)} \\
& -\frac{\pi^{3} i}{3} h_{1} h_{1} h_{2}^{(2)} h_{0}^{(4)}+\frac{4 \pi^{4}}{9} h_{1} h_{1} h_{2} h_{0}^{(4)} h_{0}^{(4)} \\
& +\frac{4 \pi^{5} i}{27} h_{1} h_{1} h_{1} h_{1}^{(2)} h_{0}^{(4)} h_{0}^{(4)}-\frac{2 \pi^{6}}{81} h_{1} h_{1} h_{1} h_{1} h_{0}^{(4)} h_{0}^{(4)} h_{0}^{(4)} \\
& -\frac{2 \pi^{2}}{3} h_{1} h_{2} h_{1}^{(4)}-\frac{5 \pi^{3} i}{18} h_{1} h_{1} h_{1}^{(2)} h_{1}^{(4)}+\frac{\pi^{4}}{6} h_{1} h_{1} h_{1} h_{0}^{(4)} h_{1}^{(4)} \\
& -\frac{\pi^{2}}{6} h_{1} h_{1} h_{2}^{(4)}+\frac{\pi^{3} i}{3} h_{1} h_{1} h_{2} h_{0}^{(6)}-\frac{\pi^{4}}{9} h_{1} h_{1} h_{1} h_{1}^{(2)} h_{0}^{(6)} \\
& \left.-\frac{\pi^{5} i}{18} h_{1} h_{1} h_{1} h_{1} h_{0}^{(4)} h_{0}^{(6)}+\frac{\pi^{3} i}{18} h_{1} h_{1} h_{1} h_{1}^{(6)}+\frac{\pi^{4}}{216} h_{1} h_{1} h_{1} h_{1} h_{0}^{(8)}\right)
\end{aligned}
$$

...

(To determine $f_{k}$ for a given $k$, it is in fact enough to let $m=k$ in (3.3) rather than taking the limit $m \rightarrow \infty$.) Assigning a formal weight $-4+2 n$ and a charge $k$ to $h_{k}^{(n)}$, we see that the general structure is that $f_{k}$ equals $\exp \left(\frac{k \pi i}{3} h_{0}^{(2)}\right)$ times a differential polynomial in $h$ of formal weight -4 and charge $k$ involving only even derivatives. The 'upper triangular' 
structure of these equations makes them easy to invert:

$$
\begin{aligned}
h_{0}= & f_{0} \\
h_{1}= & \exp \left(-\frac{\pi i}{3} f_{0}^{(2)}\right) f_{1} \\
h_{2}= & \exp \left(-\frac{2 \pi i}{3} f_{0}^{(2)}\right) \\
& \times\left(f_{2}-\frac{\pi i}{3} f_{1}^{(2)} f_{1}-\frac{2 \pi^{2}}{9} f_{0}^{(3)} f_{1}^{(1)} f_{1}+\frac{\pi^{2}}{18} f_{0}^{(4)} f_{1} f_{1}+\frac{\pi^{3} i}{27} f_{0}^{(3)} f_{0}^{(3)} f_{1} f_{1}\right)
\end{aligned}
$$

\section{The singularity structure}

Next, we need some facts about the singularities of the functions $f_{k}$. These occur at $T=i$, where the gauge group is enlarged and additional multiplets become massless. As $T \rightarrow i, f_{0}^{(2)}(T)$ diverges as $-\frac{8}{2 \pi i} \log (T-i)$ [6] [19] 20]. Furthermore, to recover the results of [21] in the limit when the string tension becomes large, $f_{k}(T)$ for $k>0$ must have a pole of order $4 k-2$ at $T=i$ [9]. Finally, it follows from (2.7) that $f_{0}^{(2)}$ diverges as $4 T$ as $T \rightarrow i \infty$. From the general form of the functions $h_{k}$ (3.7), we then conclude that their singularities are given by

$$
\begin{aligned}
& h_{k}(T) \sim(T-i)^{2-8 k / 3}, \quad T \rightarrow i \\
& h_{k}(T) \sim q_{1}^{-2 k / 3}, \quad T \rightarrow i \infty .
\end{aligned}
$$

From the fact that $f_{0}^{(5)}$ is a modular form of weight 6 and the above singularity structure, it can be determined [7] [8] as

$$
f_{0}^{(5)}=(2 \pi i)^{2} \frac{18 E_{4}^{6}-23 E_{4}^{3} E_{6}^{2}+5 E_{6}^{4}}{9 E_{6}^{3}}
$$

where the Eisenstein series of weight 4 and 6 are defined as

$$
\begin{aligned}
& E_{4}(T)=1+240 \sum_{j=1}^{\infty} \frac{j^{3} q_{1}^{j}}{1-q_{1}^{j}} \\
& E_{6}(T)=1-504 \sum_{j=1}^{\infty} \frac{j^{5} q_{1}^{j}}{1-q_{1}^{j}}
\end{aligned}
$$


for $q_{1}=\exp 2 \pi i T$. They have simple zeros at $T=\exp (\pi i / 3)$ and at $T=i$ respectively. Integrating $f_{0}^{(5)}$ five times gives $f_{0}$; all integration constants except one are determined by imposing (2.7):

$$
f_{0}(T)=\text { constant }-\frac{13}{6} T-T^{2}+\frac{2}{3} T^{3}+\mathcal{O}\left(q_{1}\right) .
$$

From (3.4) and (4.1), we conclude that for $k>0$

$$
h_{k}=(2 \pi i)^{-3} \frac{P_{24 k-16}}{\eta^{16 k} E_{6}^{8 k / 3-2}},
$$

where the $\eta$ invariant is defined as

$$
\eta(T)=q_{1}^{1 / 24} \prod_{j=1}^{\infty}\left(1-q_{1}^{j}\right)
$$

and $P_{24 k-16}$ is a holomorphic modular form of weight $24 k-16$. As such, $P_{24 k-16}$ must be of the form

$$
P_{24 k-16}=\sum_{n=1}^{2 k-1} p_{k, n} E_{4}^{3 n-1} E_{6}^{4 k-2 n-2},
$$

for some constants $p_{k, 1}, \ldots, p_{k, 2 k-1}$.

\section{The exchange symmetry}

To determine the forms $P_{24 k-16}$ in (4.5), we consider the Yukawa coupling $\left(\frac{\partial}{\partial T}\right)^{3} f$, which may be written in the form [22]

$$
\frac{\partial^{3}}{\partial T^{3}} f=4+\sum_{k=0}^{\infty} \sum_{j=1}^{\infty} j^{3} n_{j, k} \frac{q_{1}^{j} q_{2}^{k}}{1-q_{1}^{j} q_{2}^{k}} .
$$

For fixed $k>0$, the $S \leftrightarrow T$ exchange symmetry [5] [11] [12] amounts to $2 k-1$ constraints on the instantonnumbers $n_{j, k}$ in (5.1):

$$
n_{j, k}=\left\{\begin{array}{cc}
0 & , \quad 1 \leq j \leq k-1 \\
n_{j, j-k} & , \quad k \leq j \leq 2 k-1
\end{array} .\right.
$$

The $h_{k}$ can now be determined recursively: Given $h_{1}, \ldots, h_{k-1}$, the constraints (5.2) determine the constants $p_{k, 1}, \ldots, p_{k, 2 k-1}$ in (4.7) and thus, by (4.5), $h_{k}$. Explicitly, the first 
few $h_{k}$ 's are given by

$$
\begin{aligned}
h_{1}= & (2 \pi i)^{-3} \eta^{-16} E_{6}^{-2 / 3} 2 E_{4}^{2} \\
h_{2}= & (2 \pi i)^{-3} 2^{-4} 3^{-3} \eta^{-32} E_{6}^{-10 / 3}\left(-89 E_{4}^{8}-53 E_{4}^{5} E_{6}^{2}+122 E_{4}^{2} E_{6}^{4}\right) \\
h_{3}=(2 \pi i)^{-3} 2^{-5} 3^{-7} \eta^{-48} E_{6}^{-6} & \quad \times\left(20367 E_{4}^{14}-38052 E_{4}^{11} E_{6}^{2}+18898 E_{4}^{8} E_{6}^{4}-6260 E_{4}^{5} E_{6}^{6}+3895 E_{4}^{2} E_{6}^{8}\right) \\
& \quad(2 \pi i)^{-3} 2^{-13} 3^{-10} \eta^{-64} E_{6}^{-26 / 3} \\
h_{4}= & \left(216412213 E_{4}^{20}-793763223 E_{4}^{17} E_{6}^{2}+1110594390 E_{4}^{14} E_{6}^{4}\right. \\
& \quad-711685317 E_{4}^{11} E_{6}^{6}+217366407 E_{4}^{8} E_{6}^{8}-18944802 E_{4}^{5} E_{6}^{10} \\
& \left.+5991276 E_{4}^{2} E_{6}^{12}\right)
\end{aligned}
$$

(We have also calculated $h_{5}, h_{6}$ and $h_{7}$.) Inserting this result in (3.6) gives the functions $f_{k}$ in the prepotential. The instanton expansion (5.1) then gives the instantonnumbers $n_{j, k}$, which count rational curves in the Calabi-Yau manifold:

$$
\mathrm{j}=0 \quad \mathrm{k}=1 \quad \mathrm{k}=2 \ldots
$$

0

1

2

3

4

5

6

6

$7 \quad 274856132550917568$

0

2496

223752

38637504

9100224984

2557481027520

805628041231176
199399229066445715968
331025557765003648

2

2496

1941264

1327392512

861202986072

540194037151104

5143228729806654496

5458385566105678112256

These numbers agree with the results of [5].

Thus, we have established the claim that the monodromy, singularity structure, and $S \leftrightarrow T$ exchange symmetry are sufficient to determine the prepotential exactly in the region where the series converges.

\section{Acknowledgements}


We would like to thank J. Harvey for participation at the beginning of this project and for discussions. We also thank P. Berglund, A. Losev, D. Morrison, N. Nekrasov, R. Plesser, and S. Shatashvili for useful correspondence and discussions. This research is supported by DOE grant DE-FG02-92ER40704, and by a Presidential Young Investigator Award. GM also thanks the Rutgers high energy theory group for hospitality during the completion of this paper. 


\section{References}

[1] S. Ferrara, J. A. Harvey, A. Strominger and C. Vafa, "Second-Quantized Mirror Symmetry", Phys. Lett. B361 (1995) 59, hep-th/9505162.

[2] S. Kachru and C. Vafa, "Exact Results for $\mathrm{N}=2$ Compactifications of Heterotic Strings", Nucl. Phys. B450 (1995) 69, hep-th/9505105.

[3] P. S. Aspinwall and J. Louis, "On the Ubiquity of K3 Fibrations in String Duality", hep-th/9510234.

[4] S. Hosono, A. Klemm, S. Theisen and S.T. Yau, "Mirror Symmetry, Mirror Map and Applications to Calabi-Yau Hypersurfaces", Comm. Math. Phys. 167 (1995) 301, hep-th/9308122;

S. Hosono, A. Klemm, S. Theisen and S.T. Yau, "Mirror Symmetry, Mirror Map, and Applications to Complete Intersection Calabi-Yau Spaces", Nucl. Phys. B433 (1995) 501, hep-th/9406055.

[5] P. Candelas, X. De la Ossa, A. Font, S. Katz and D. Morrison, "Mirror Symmetry for Two Parameter Models - I", Nucl. Phys. B416 (1994) 481, hep-th/9308083.

[6] B. de Wit, V. Kaplunovsky, J. Louis and D. Lüst, "Perturbative Couplings of Vector Multiplets in N=2 Heterotic String Vacua", Nucl. Phys. B451 (1995) 53, hepth/9504006.

[7] V. Kaplunovsky, J. Louis and S. Theisen, "Aspects of Duality in N=2 String Vacua", Phys. Lett. B357 (1995) 71, hep-th/9506110.

[8] I. Antoniadis, S. Ferrara, E. Gava, K.S. Narain and T.R. Taylor, "Duality Symmetries in N=2 Heterotic Superstring", hep-th/9510079.

[9] S. Kachru, A. Klemm, W. Lerche, P. Mayr and C. Vafa, "Nonperturbative Results on the Point Particle Limit of N=2 Heterotic String Compactifications", hepth/9508155.

[10] B. H. Lian and S.-T. Yau, "Mirror Maps, Modular Relations and Hypergeometric Series. 2", hep-th/9507153.

[11] A. Klemm, W. Lerche, and P. Mayr, "K3-Fibrations and Heterotic-Type II String Duality", Phys. Lett. B357 (1995) 313, hep-th/9506112.

[12] G. L. Cardoso, G. Curio, D. Lüst, T. Mohaupt and S.-J. Rey, "BPS Spectra and NonPerturbative Gravitational Couplings in N=2, 4 Supersymmetric String Theories", hep-th/9512129.

[13] V. A. Gritsenko and V. V. Nikulin, "K3 surfaces, Lorentzian Kac-Moody algebras and Mirror Symmetry", alg-geom/9510008.

[14] J. Jorgenson and A. Todorov, "Analytic Discriminants for Manifolds with Canonical Class Zero", Yale preprint ;

J. Jorgenson and A. Todorov, "Enriques surfaces, Analytic discriminants, and Borcherds' $\Phi$ function", Yale preprint ; 
J. Jorgenson and A. Todorov, "A Conjectured Analogoue of Dedekind's Eta Function for K3 Surfaces", Math. Res. Lett. 2 (1995) 359.

[15] J. A. Harvey and G. Moore, "Algebras, BPS States, and Strings", hep-th/9510182.

[16] T. Kawai, " $N=2$ heterotic string threshold correction, $K 3$ surface and generalized Kac-Moody superalgebra", hep-th/9512046.

[17] B. de Wit and A. van Proeyen, Nucl. Phys. B245 (1984) 89.

[18] A. Ceresole, R. D'Auria and S. Ferrara, Phys. Lett. B339 (1994) 71, hep-th/9408036.

[19] I. Antoniadis, S. Ferrara, E. Gava, K.S. Narain and T.R. Taylor, "Perturbative Prepotential and Monodromies in N=2 Heterotic Superstring", Nucl. Phys. B447 (1995) 35 , hep-th/9504034.

[20] G. L. Cardoso, D. Lüst and T. Mohaupt, "Threshold Corrections and Symmetry Enhancement in String Compactifications", Nucl. Phys. B450 (1995) 115, hepth/9412209.

[21] N. Seiberg and E. Witten, "Electric-Magnetic Duality, Monopole Condensation, and Confinement in $N=2$ Supersymmetric Yang-Mills Theory", Nucl. Phys. B426 (1994) 19, hep-th/9407087.

[22] P. S. Aspinwall and D. R. Morrison, "Topological Field Theory and Rational Curves", Comm. Math. Phys. 151 (1993) 245, hep-th/9110048. 